\title{
A MEAN ERGODIC THEOREM IN BANACH SPACES
}

\author{
TAKESHI YOSHIMOTO
}

\begin{abstract}
Let $\Gamma=\left\{U_{t}: t \in \Lambda\right\}\left(\Lambda=\mathbf{Z}^{+}-\{0\}\right.$ or $\left.\mathbf{R}^{+}-\{0\}\right)$ be a commuting family of nonexpansive affine operators in a Banach space $X$ satisfying the following conditions:
\end{abstract}

(i) there is a function $M(x \mid \Gamma) \geq 0$ defined on $X$ such that

$$
\left\|U_{t+s} x-U_{t} U_{s} x\right\| \leq M(x \mid \Gamma) \quad(s, t \in \Lambda, x \in X),
$$

(ii) $\sup \left\{t^{-1}\left\|U_{t} x\right\|: t \in \Lambda, t \geq 1\right\}=K(x \mid \Gamma)<\infty(x \in X)$.

Then it is proved that if $\left\{t^{-1} U_{t} x: t \in \Lambda\right\}$ is relatively compact for $x \in X$, the limit $X-\lim _{t \rightarrow \infty} t^{-1} U_{t} x=\bar{x}$ exists in $X$ and $\overline{U_{t} x}=\bar{x}(t \in \Lambda)$.

1. Introduction. Let $(X,\|\cdot\|)$ be a general Banach space. $\mathbf{Z}^{+}$is the set of nonnegative integers and $\mathbf{R}^{+}$is the set of nonnegative real numbers. In this paper, $\Lambda$ will be used for denoting either $\mathbf{Z}^{+}-\{0\}$ or $\mathbf{R}^{+}-\{0\}$. For $x, y \in X$ and $\alpha \in \mathbf{R}^{+}$, we write

$$
x \approx y \quad(\bmod \alpha) \quad \text { if }\|x-y\| \leq \alpha .
$$

Let $\Gamma=\left\{U_{t}: t \in \Lambda\right\}$ be a commuting family of operators on $X$ to $X$. The family $\Gamma$ is said to have a modulus function $M(x \mid \Gamma)$ if there exists a function $M(x \mid \Gamma) \geq 0$ defined on $X$ such that for each $x \in X$

$$
U_{t+s} x \approx U_{t} U_{s} x \quad(\bmod M(x \mid \Gamma)), \quad s, t \in \Lambda .
$$

For the family $\Gamma$ we define the following conditions:

(I) $\Gamma$ is nonexpansive, i.e.,

$$
\left\|U_{t} x-U_{t} y\right\| \leq\|x-y\|, \quad x, y \in X, t \in \Lambda .
$$

(II) $\Gamma$ has a modulus function $M(\cdot \mid \Gamma)$ defined on $X$.

(III) There are functions $H_{t}(x, y \mid \Gamma) \geq 0, t \in \Lambda$, defined on $X \times X$ with

$$
\lim _{t \rightarrow \infty} \sup _{s \in \Lambda} H_{t}\left(U_{s} x, x \mid \Gamma\right)=0 \quad(x \in X)
$$

such that for some $\delta \in \mathbf{R}^{+}$,

$$
U_{t}\{\alpha x+(1-\alpha) y\} \approx \alpha U_{t} x+(1-\alpha) U_{t} y \quad\left(\bmod H_{t}(x, y \mid \Gamma)+\delta\right)
$$

$(x, y \in X, t \in \Lambda)$ uniformly in $\alpha$ for $0 \leq \alpha \leq 1$.

(IV) $\sup \left\{t^{-1}\left\|U_{t} x\right\|: t \in \Lambda, t \geq 1\right\}=K(x \mid \Gamma)<\infty(x \in X)$.

Conditions (I) -(IV) are always satisfied for semigroups of linear contractions, or more generally, for uniformly bounded semigroups of nonexpansive affine operators on $X$.

We prove the following theorem.

Received by the editors October 10, 1985.

1980 Mathematics Subject Classification (1985 Revision). Primary 47A35. 
THEOREM. Let $\Gamma=\left\{U_{t}: t \in \Lambda\right\}$ be a commuting family of operators on $X$ satisfying conditions (I)-(IV). If for $x \in X$, the set $\left\{t^{-1} U_{t} x: t \in \Lambda\right\}$ is relatively compact, then there exists an $\bar{x} \in X$ such that

$$
X-\lim _{t \rightarrow \infty} t^{-1} U_{t} x=\bar{x} \quad \text { and } \quad \overline{U_{t} x}=\bar{x}, \quad t \in \Lambda .
$$

In [4], Rodé proved that if $\left\{P^{n}: n \in \mathbf{Z}^{+}\right\}$is the discrete semigroup of convex Markov operators on a Banach lattice $E$ and if $\left\{n^{-1} P^{n} 0: n \in \mathbf{Z}^{+}-\{0\}\right\}$ is relatively compact, then $n^{-1} P^{n} 0$ converges strongly in $E$ as $n \rightarrow \infty$. Here the convex Markov operator $P$ is supposed to be convex, monotone, and nonexpansive. The case for a general Banach space seems to have been unnoticed by Rodé. We know of no such result in general Banach spaces in the literature. Our theorem provides conditions for the validity of the mean convergence of $t^{-1} U_{t} x$ for each $x$ in a general Banach space $X$.

2. Proof of the Theorem. We note that $\left\{t^{-1} U_{t} x: t \in \Lambda\right\}$ is sequentially compact as $t \rightarrow \infty$. So, there exist an $\bar{x} \in X$ and a sequence $\left\{s_{i}\right\} \subset \Lambda$ with $1<s_{1}<s_{2}<\cdots \rightarrow \infty$ such that $\lim _{i \rightarrow \infty}\left\|s_{i}^{-1} U_{s_{i}} x-\bar{x}\right\|=0$. According to conditions (I)-(II),

$$
\left\|\frac{1}{s_{i}} U_{s_{i}+t} x-\bar{x}\right\| \leq \frac{1}{s_{i}}\left\{M(x \mid \Gamma)+\left\|U_{t} x-x\right\|\right\}+\left\|\frac{1}{s_{i}} U_{s_{i}} x-\bar{x}\right\|,
$$

which implies that $\lim _{i \rightarrow \infty}\left\|s_{i}^{-1} U_{s_{i}+t} x-\bar{x}\right\|=0$ for each $t \in \Lambda$. Let $s$ be any element of $\Lambda$. Then for $s_{i}>s$, it follows by conditions (II)-(III) that

$$
\begin{aligned}
U_{t}\left\{\frac{s}{s_{i}} U_{s_{i}} x+\right. & \left.\left(1-\frac{s}{s_{i}}\right) y\right\} \approx \frac{s}{s_{i}} U_{s_{i}+t} x+\left(1-\frac{s}{s_{i}}\right) U_{t} y \\
& \left(\bmod \left(\frac{s}{s_{i}} M(x \mid \Gamma)+H_{t}\left(U_{s_{i}} x, y \mid \Gamma\right)+\delta\right)\right), \quad y \in X, t \in \Lambda .
\end{aligned}
$$

We thus have

$$
\begin{aligned}
\left\|U_{t}(s \bar{x}+y)-\left(s \bar{x}+U_{t} y\right)\right\| \leq & s\left\{\left\|\frac{1}{s_{i}} U_{s_{i}} x-\bar{x}\right\|+\left\|\frac{1}{s_{i}} U_{s_{i}+t} x-\bar{x}\right\|\right\} \\
& +\frac{s}{s_{i}}\left\{\|y\|+\left\|U_{t} y\right\|+M(x \mid \Gamma)\right\}+H_{t}\left(U_{s_{i}} x, y \mid \Gamma\right)+\delta
\end{aligned}
$$

for any $y \in X$. Hence, letting $i \rightarrow \infty$ in the above gives

$$
U_{t}(s \bar{x}+x) \approx s \bar{x}+U_{t} x \quad\left(\bmod \left(\sup _{r \in \Lambda} H_{t}\left(U_{r} x, x \mid \Gamma\right)+\delta\right)\right)
$$

for any $x, t \in \Lambda$. Now define, for $s, t \in \Lambda$,

$$
Z_{s}(x)=U_{s} x-s \bar{x}, \quad Z_{s}^{t}(x)=U_{s+t} x-U_{t}(s \bar{x}+x) .
$$

We obtain $\left\|Z_{s}^{t}(x)\right\| \leq M(x \mid \Gamma)+\left\|Z_{s}(x)-x\right\|$, and therefore,

$$
\begin{aligned}
\left\|U_{s+t} x-\left(U_{s} x+U_{t} x\right)\right\| & \leq\left\|U_{t}(s \bar{x}+x)-\left(s \bar{x}+U_{t} x\right)\right\|+\left\|Z_{s}^{t}(x)\right\|+\left\|Z_{s}(x)\right\| \\
& \leq M(x \mid \Gamma)+\|x\|+2\left\|Z_{s}(x)\right\|+\sup _{r \in \Lambda} H_{t}\left(U_{r} x, x \mid \Gamma\right)+\delta
\end{aligned}
$$

for any $s, t \in \Lambda$. For any given $\varepsilon>0$, choose a number $i_{0}$ such that

$$
s_{i_{0}}^{-1}\left\|Z_{s_{i_{0}}}(x)\right\|<\frac{\varepsilon}{9}, \quad s_{i_{0}}^{-1} \delta<\frac{\varepsilon}{3}, \quad s_{i_{0}}^{-1}\{\|x\|+M(x \mid \Gamma)\}<\frac{\varepsilon}{3} .
$$


Put $s_{0}=s_{i_{0}}$ and let $\beta=t-n s_{0}$ for sufficiently large $t \geq s_{0}^{2}+s_{0}+1$, where $n=\left[s_{0}^{-1}(t-1)\right]$ (the integer part of $s_{0}^{-1}(t-1)$ ). Clearly $n \geq s_{0}$ and $1 \leq \beta<s_{0}+1$. Then it follows that

$$
\begin{aligned}
\left\|U_{t} x-n U_{s_{0}} x-U_{\beta} x\right\| \leq & n\left\{M(x \mid \Gamma)+\|x\|+2\left\|Z_{s_{0}}(x)\right\|\right\} \\
& +\sum_{k=1}^{n} \sup _{r \in \Lambda} H_{(k-1) s_{0}+\beta}\left(U_{r} x, x \mid \Gamma\right)+n \delta .
\end{aligned}
$$

Therefore we have

$$
\begin{aligned}
\varlimsup_{t \rightarrow \infty}\left\|\frac{1}{t} U_{t} x-\bar{x}\right\| \leq & \varlimsup_{t \rightarrow \infty} \frac{n}{t}\left\{M(x \mid \Gamma)+\|x\|+3\left\|Z_{s_{0}}(x)\right\|+\delta\right\} \\
& +\varlimsup_{t \rightarrow \infty} \frac{s_{0}+1}{t}\{\|\bar{x}\|+K(x \mid \Gamma)\} \\
& +\varlimsup_{t \rightarrow \infty} \frac{1}{t} \sum_{k=1}^{n} \sup _{r \in \Lambda} H_{(k-1) s_{0}+\beta}\left(U_{r} x, x \mid \Gamma\right) \\
\leq & \frac{1}{s_{0}}\left\{M(x \mid \Gamma)+\|x\|+3\left\|Z_{s_{0}}(x)\right\|+\delta\right\}<\varepsilon .
\end{aligned}
$$

Finally, to see that $\overline{U_{t} x}=\bar{x}(t \in \Lambda)$, it is enough to note that for any $t$

$$
\left\|\frac{1}{s} U_{s} U_{t} x-\bar{x}\right\| \leq \frac{1}{s} M(x \mid \Gamma)+\left\|\frac{s+t}{s} \frac{1}{s+t} U_{s+t} x-\bar{x}\right\|,
$$

which tends to zero as $s \rightarrow \infty$. Hence the proof is complete.

3. Applications. The method of proof in $\S 2$ can be applied to the study of ergodic behaviors of additive processes.

COROLLARY 1. Let $\mathfrak{S}=\left\{T_{t}: t \in \Lambda\right\}$ be a commuting family of linear contractions on $X$ with a modulus function $M(x \mid \mathfrak{S})$. Let $\left\{F_{t}: t \in \Lambda\right\}$ be an $M(\alpha)$-additive process in $X$ with respect to $\mathfrak{S}$, i.e.,

(i) $F_{t+s} \approx F_{t}+T_{t} F_{s}(\bmod \alpha), s, t \in \Lambda(\alpha \geq 0)$,

(ii) $\sup \left\{t^{-1}\left\|F_{t}\right\|: t \in \Lambda, t \geq 1\right\}=K<\infty$.

If $\left\{t^{-1} F_{t}: t \in \Lambda\right\}$ is relatively compact, then there exists a $\mathfrak{S}$-invariant $f \in X$ such that $\lim _{t \rightarrow \infty} t^{-1} F_{t}=f$ strongly in $X$.

ProOF. Define

$$
U_{t} x=T_{t} x+F_{t}, \quad x \in X, t \in \Lambda .
$$

Then the family $\Gamma=\left\{U_{t}: t \in \Lambda\right\}$ is commutative and satisfies conditions (I)(IV), where $M(x \mid \Gamma) \equiv M(x \mid \mathfrak{S})+\alpha, H_{t}(x, y \mid \Gamma) \equiv 0$, and $K(x \mid \Gamma) \equiv K+\|x\|$. Since $\left\|T_{t}\right\| \leq 1, t \in \Lambda$, the relative compactness of $\left\{t^{-1} F_{t}: t \in \Lambda\right\}$ implies that of $\left\{t^{-1} U_{t} x: t \in \Lambda\right\}$ for $x \in X$. From this it follows by the theorem that there exists an $f \in X$ such that $\lim _{t \rightarrow \infty} t^{-1} F_{t}=f$ strongly in $X$. Furthermore, it is easily seen that $\lim _{t \rightarrow \infty} t^{-1}\left\|F_{t+u}-F_{t}\right\|=0$ for any $u \in \Lambda$, and so, $\lim _{t \rightarrow \infty} t^{-1}\left\|F_{t}-T_{u} F_{t}\right\|=0$. Therefore we have

$$
\left\|f-T_{u} f\right\| \leq \lim _{t \rightarrow \infty}\left\{2\left\|t^{-1} F_{t}-f\right\|+t^{-1}\left\|F_{t}-T_{u} F_{t}\right\|\right\}=0
$$

that is, $f$ is S-invariant. 
COROLLARY 2. Let $\left\{U_{t}: t \in \Lambda\right\}$ be a semigroup of nonexpansive affine operators on $X$ such that $\sup \left\{t^{-1}\left\|U_{t} x\right\|: t \in \Lambda, t \geq 1\right\}=K(x)<\infty$ for $x \in X$. If for $x \in X,\left\{t^{-1} U_{t} x: t \in \Lambda\right\}$ is relatively compact, then there exists an $\bar{x} \in X$ such that $\lim _{t \rightarrow \infty} t^{-1} U_{t} x=\bar{x}$ strongly in $X$ and $\overline{U_{t} x}=\bar{x}(t \in \Lambda)$.

EXAMPLE 1. In the special case of affine operators,

$$
U x=T(x-\xi)+\eta, \quad x \in X,
$$

with a linear contraction $T$ on $X$ and some $\xi, \eta \in X$, we obtain

$$
\frac{1}{n} U^{n} x=\frac{1}{n} T^{n}(x-\xi)+\frac{1}{n} \sum_{k=0}^{n-1} T^{k} \eta-\frac{1}{n} \sum_{k=0}^{n-1} T^{k} \xi+\frac{\xi}{n} .
$$

Thus if $x=\xi=0$, then

$$
\frac{1}{n} U^{n} 0=\frac{1}{n} \sum_{k=0}^{n-1} T^{k} \eta \quad \text { (the Cesàro mean). }
$$

EXAMPLE 2. Let $\left\{T_{t}: t \in \mathbf{R}^{+}-\{0\}\right\}$ be a strongly continuous semigroup of linear contractions on $X$ and let $\left\{F_{t}: t \in \mathbf{R}^{+}-\{0\}\right\}$ be an additive process in $X$ with respect to $\left\{T_{t}\right\}$. Define

$$
U_{t} x=T_{t} x+F_{t}, \quad x \in X, t \in \mathbf{R}^{+}-\{0\} .
$$

It is easily seen that $\left\{U_{t}: t \in \mathbf{R}^{+}-\{0\}\right\}$ is a semigroup of nonexpansive affine operators on $X$ such that $\sup \left\{t^{-1}\left\|U_{t} x\right\|: t \geq 1\right\}<\infty(x \in X)$. If $X$ is reflexive, then there exists a $\xi \in X$ such that $F_{t}=\int_{0}^{t} T_{u} \xi d u, t>0[1$, Theorem 10]. In this case, the set $C_{\xi}=\left\{t^{-1} \int_{0}^{t} T_{u} \xi d u: t>0\right\}$ is weakly sequentially compact as $t \rightarrow \infty$, and so by the mean ergodic theorem, $C_{\xi}$ is also (strongly) sequentially compact. From this it follows that for each $x \in X,\left\{t^{-1} U_{t} x: t>0\right\}$ is sequentially compact. Hence $\lim _{t \rightarrow \infty} t^{-1} U_{t} x(x \in X)$ exists strongly in $X$.

EXAMPLE 3. Let $G$ be a compact metric space and let $\left\{\varphi_{t}: t \in \mathbf{R}^{+}-\{0\}\right\}$ be a nonexpansive semiflow on $G$. Assume that there is given an equicontinuous additive process $\left\{F_{t}: t \in \mathbf{R}^{+}-\{0\}\right\}$ in $C(G)$ with respect to $\left\{\varphi_{t}\right\}$, i.e., $F_{t+s}=F_{t}+F_{s}$ 。 $\varphi_{t}(s, t>0), \sup \left\{t^{-1}\left\|F_{t}\right\|: t>0\right\}<\infty$, and to every $\varepsilon>0$ and every $u \in G$ there corresponds a neighborhood $V(u)$ of $u$ such that $\sup _{t>0} \sup _{v \in V(u)}\left|F_{t}(u)-F_{t}(v)\right|<$ $\varepsilon$. We define

$$
U_{t} f=f \circ \varphi_{t}+F_{t}, \quad f \in C(G), t \in \mathbf{R}^{+}-\{0\} .
$$

Then $\left\{U_{t}: t \in \mathbf{R}^{+}-\{0\}\right\}$ turns out to be a semigroup of nonexpansive affine operators on $C(G)$ such that $\sup \left\{t^{-1}\left\|U_{t} f\right\|: t \geq 1\right\}<\infty$ for $f \in C(G)$. Using the Arzelà-Ascoli theorem we see that for $f \in C(G),\left\{t^{-1} U_{t} f: t \geq 1\right\}$ is sequentially compact as $t \rightarrow \infty$. Hence, $\lim _{t \rightarrow \infty} t^{-1} U_{t} f(f \in C(G))$ exists strongly in $C(G)$.

\section{REFERENCES}

1. M. A. Akcoglu and U. Krengel, A differentiation theorem in $L_{p}$, Math. Z. 169 (1979), 31-40.

2. N. Dunford, $A$ mean ergodic theorem, Duke Math. J. 5 (1939), 635-646.

3. N. Dunford and J. T. Schwartz, Linear operators, Part 1, Interscience, New York, 1958.

4. G. Rodé, An ergodic theorem for convex operators, Arch. Math. 40 (1983), 447-451.

Department of MATHEMATICS, Toyo University, SAITAMA, JAPAN 